\title{
Bacteriological Profile and Antibiotic Sensitivity Pattern of Neonatal Sepsis in a Tertiary Care Hospital
}

\author{
Pavneet Kaur* and Sarbjeet Sharma
}

Department of Microbiology, Sri Guru Ram Das Institute of Medical Sciences and Research, Amritsar, India

*Corresponding author

\begin{tabular}{|l|}
\hline Ke y w o r d s \\
Neonate, Blood \\
culture, ESBL, \\
AmpC, GNB \\
\hline Article Info \\
\hline $\begin{array}{l}\text { Accepted: } \\
\text { 26 October } 2018 \\
\text { Available Online: } \\
\text { 10 November } 2018\end{array}$ \\
\hline
\end{tabular}

A B S T R A C T

India accounts for $30 \%$ of neonatal deaths globally. Bacterial sepsis is a significant cause of morbidity and mortality in newborns. The study helps to make antibiotic policy in neonatal sepsis. The main objective is to study the incidence of multidrug resistant gram negative and gram positive organisms causing neonatal septicemia and their antibiotic sensitivity pattern. The study was conducted in the Department of Microbiology over a period of one year. Sample of blood was collected under aseptic precautions and processed by standard techniques. Microorganisms were identified by Gram staining, standard biochemical tests and appropriate antibiograms. The common microorganisms responsible for neonatal sepsis were identified, and the resistant strains were studied. After identification and antibiotic susceptibility testing, beta-lactamases were detected as per CLSI guidelines. In 233 blood cultures $18.9 \%$ (44/233) culture positivity was seen. Out of them, $31(70.5 \%)$ were Gram negative and 13(29.5\%) were Gram positive. Klebsiella pneumoniae subspecies pneumoniae $(45.5 \%)$ was the most common isolate. ESBL producers were maximum (54.8\%.) $25.8 \%$ of the isolates were positive for AmpC production. The diverse microbiological pattern of neonatal septicaemia demands the need for review of neonatal sepsis. The evaluation of the pathogens and their antibiotic susceptibility is a relevant guide in the antibiotic therapy.

\section{Introduction}

Neonates with low immunity, always need prolonged hospitalization which is a risk factor of post-infectious complications. (Adams-Chapman and Stoll, 2002) It is estimated that about 5 million neonatal deaths occur in a year out of which $98 \%$ occur in developing countries. (WHO, 1996) Medical advancements of the last twenty years have increased the survival rate of neonates. Most common causative bacteria in developed countries includes Coagulase-negative staphylococcus and group B Streptococcus while in developing countries are E. coli, Klebsiella, Enterobacter etc. (Waheed et al., 2003) Klebsiella and Enterobacter species are often associated with the production of extended-spectrum beta-lactamase (ESBL) among the multiresistant Gram-negative bacteria. (Karunasekera and Pathirana, 1999) The control of these hospital acquired infections has been a challenging task. (Goldmann et al., 1983) Spectrum of 
multiresistant organisms causing neonatal sepsis is variable over time and across countries. Thus periodic surveillance is essential.

\section{Materials and Methods}

A prospective study was conducted over a period of one year in the department of Microbiology in a tertiary care centre. Blood was collected from 233 cases of neonatal septicemia from NICU, under aseptic precautions and processed by standard techniques. Standard microbiological techniques were used for identification the organisms grown in pure culture (Collee $e t$ al., 1996). Antibiotic susceptibility testing of Gram-negative isolates was done on MuellerHinton agar plates by the Clinical and Laboratory Standards Institute (CLSI)recommended Kirby-Bauer disc diffusion method with discs of Gentamicin $(10 \mu \mathrm{g})$, Amikacin $\quad(30 \quad \mu \mathrm{g})$, third-generation Cephalosporin $(30 \mu \mathrm{g})$, Ciprofloxacin $(5 \mu \mathrm{g})$, Piperacillin-Tazobactam $(100+10 \mu \mathrm{g})$ and Meropenem $(10 \mu \mathrm{g})$. (Clinical and laboratory Standard Institute Guidelines, 2013; Lalitha, 2011) The isolates were tested for ESBL production using double disc approximation test and CLSI phenotypic confirmatory test. $E$. coli ATCC 25922 and Klebsiella pneumoniae ATCC 700603 were used as the negative and positive controls. Amp-C $\beta$-lactamases producers were screened by using reduced cefoxitin susceptibility and production was confirmed by modified three dimensional test. An indentation or a flattening of the zone of inhibition indicated AmpC production (Shahid et al., 2004).

Antibiotic sensitivity tests of staphylococcal isolates were done in Mueller-Hinton agar plates by Kirby-Bauer disc diffusion method. Antibiotics used were Gentamicin $(10 \mu \mathrm{g})$, Erythromycin $(15 \mu \mathrm{g})$, Cefoxitin $(30 \mu \mathrm{g})$, Amikacin $(30 \mu \mathrm{g})$, Rifampicin $(5 \mu \mathrm{g})$,
Clindamycin $(2 \mu \mathrm{g})$, Linezolid $(30 \mu \mathrm{g})$. The control strain used was $S$. aureus ATCC 25923. The results were interpreted according to the CLSI guidelines (Clinical and laboratory Standard Institute Guidelines, 2013; Lalitha, 2011)

\section{Results and Discussion}

Out of 233 newborns with neonatal sepsis included in the study, $44(18.9 \%)$ positivity was seen. The predominant organisms responsible for septicemia were S. aureus, Klebsiella Enterobacter, Pseudomonas and Acinetobacter spp. Among the 44 isolates, 31 (70.5\%) were Gram-negative organisms and $13(29.5 \%)$ were Gram-positive organisms.

Klebsiella pneumoniae subspecies pneumoniae was the most common organism isolated $20 \quad(45.5 \%)$ followed by Staphylococcus aureus 12(27.3), E. coli 7 (15.9\%), Acinetobacter 2(4.5\%) and Pseudomonas 2 (4.5\%). Table 1 and 2 show the antibiotic sensitivity pattern of Gramnegative and Gram-positive isolates, respectively. The total prevalence of ESBL production in this study was $54.8 \%$. Among $K$. pneumoniae, $60 \%$ were ESBL producers whereas $40 \%$ were non-ESBL. $4(57.1 \%)$ out of 7 E. coli were ESBL producers. Among the 31 Gram-negative isolates, $8(25.8 \%)$ were AmpC production positive.

Among Coagulase positive staphylococci, all were methicillin sensitive (MSSA). The sensitivity of amikacin and ciprofloxacin showed sensitive trend with most of the gram negative organisms. Imepenem was maximum sensitive among all gram negative organisms. Third generation with inhibitor i.e. Pipra + tazobactam also showed good sensitivity as shown in Table 1. In case of Gram positive Vancomycin, clindamycin, cefoxitin and Linezolid, were found most effective as shown in Table 2. 
Neonatal sepsis is an invasive infection usually bacterial / fungal in origin and documented by a positive blood culture. It is the most common constituent contributing to NICU admissions in developing countries. Blood culture positivity in these cases has shown wide variation over the years. (Mondal et al., 1991) It is a leading cause of morbidity and mortality.

In the present study, blood culture was positive in $18.9 \%$. The predominant organisms isolated were Klebsiella spp and $S$. aureus which is similar to a study from (NNPD, 2000). Thus, Klebsiella spp and S.aureus are the most common causative organisms responsible for neonatal septicemia in India.

Of the 44 culture positive isolates, 20 (45.5\%) were of Klebsiella spp. The most sensitive drugs were Imipenem, Piperacillin + tazobactam and Cefoperzone +sulbactam combination.

Table.1 Sensitivity and resistance percentage of gram negative organisms to antibiotics

\begin{tabular}{|c|c|c|c|c|c|c|c|c|}
\hline Antibiotic & \multicolumn{2}{|c|}{ E coli $(7)$} & \multicolumn{2}{|c|}{ Klebsiella (20) } & \multicolumn{2}{|c|}{ Pseudomonas (2) } & \multicolumn{2}{|c|}{ Acinetobacter (2) } \\
\hline & $\mathrm{S} \%$ & $\mathrm{R} \%$ & $\mathrm{~S} \%$ & $\mathrm{R} \%$ & $\mathrm{~S} \%$ & $\mathrm{R} \%$ & $\mathrm{~S} \%$ & $\mathrm{R} \%$ \\
\hline Gentamicin & 42.9 & 57.1 & 30 & 70 & 50 & 50 & 50 & 50 \\
\hline Amikacin & 85.7 & 14.3 & 40 & 60 & 50 & 50 & 50 & 50 \\
\hline Ciprofloxacin & 71.4 & 28.6 & 60 & 40 & 100 & 0 & 50 & 50 \\
\hline Ceftriaxone & 42.9 & 57.1 & 35 & 65 & NT* & $\mathrm{NT}^{*}$ & 50 & 50 \\
\hline Imepenem & 100 & 0 & 100 & 0 & 100 & 0 & 100 & 0 \\
\hline Pipra + Tazo & 85.7 & 14.3 & 85 & 15 & 100 & 0 & 50 & 50 \\
\hline $\begin{array}{l}\text { Cefoperazone + } \\
\text { sulbactum }\end{array}$ & 71.4 & 28.6 & 85 & 15 & NT* & $\mathrm{NT}^{*}$ & 50.0 & 50.0 \\
\hline
\end{tabular}

*NT=Not tested

Table.2 Sensitivity and resistance percentage of gram positive organisms to antibiotics

\begin{tabular}{|l|c|l|l|l|}
\hline Antibiotic & \multicolumn{3}{|c|}{ S aureus(12) } & \multicolumn{2}{c|}{ CONS(1) } \\
\hline Cephalexin & S \% & R \% & S \% & R \% \\
\hline Gentamicin & 100 & 0 & 100 & 0 \\
\hline Amikacin & 75 & 25 & 100 & 0 \\
\hline Cefoxitin & 100 & 0 & 100 & 0 \\
\hline Vancomycin & 100 & 0 & 100 & 0 \\
\hline Erythromycin & 100 & 0 & NT* $^{*}$ & NT* \\
\hline Rifampicin & 66.7 & 33.3 & 100 & 0 \\
\hline Clindamycin & 100 & 0 & 100 & 0 \\
\hline Linezolid & 100 & 0 & 100 & 0 \\
\hline *NT=Not tested & 100 & 0 & NT* $^{*}$ & NT* \\
\hline
\end{tabular}


The present study outlines the importance of higher generation cephalosporin with beta lactamases inhibitor combination for empirical therapy.

In a study by Shah et al., in 2012, Gramnegative organisms were isolated in 52\% cases, Gram-positive in 45\% cases and Candida species in 3\% cases. (Shah et al., 2012) The increased incidence of sepsis by Gram-negative bacteria may be attributed to the fact that there is colonisation of Gramnegative bacteria in the skin of the neonate and the personnel of the neonatal wards.

In a study by (Abdel-Hady et al., 2008) 67\% of the Klebsiella isolates were ESBL producers which is consistent with the present study.

ESBLs production is not routinely tested in most centres resulting in the dissemination of ESBL-producing strains within and between hospitals and remains undetected for long periods. Similar to study conducted by (Jain and Mondal, 2007), a high incidence of lactamases production in Klebsiella spp was observed, which may be due to injudicious use of antibiotics. Hence, prophylactic use of antibiotics should be with caution, while therapeutic antibiotics should be specific and used for as short a period of time as possible. Rotating antibiotic regimens is suggested in conditions where the use of antibiotics is necessary.

There was no MRSA among gram positive isolates in this study. $33 \%$ of CONS were found in the present study which can be decreased by following appropriate aseptic precautions in collection of blood samples. Relevant educational programs combined with rotational antibiotic therapy may be useful in fighting against such types of infections. Considering widespread antibiotic resistance in our country, it is essential to determine the prevalence of $\beta$-lactamases producers to aid in formulating an antibiotic policy for empirical therapy in NICU.

The varying microbiological pattern of neonatal septicaemia warrants the need for periodic review of neonatal sepsis as the knowledge of the pathogens and their antibiotic susceptibility would be a useful guide in the antibiotic therapy This will also facilitate infection control interventions like hand hygiene, patient isolation and contact precautions

\section{References}

Abdel-Hady H, Hawas S, El-Daker M, ElKady R. Extended-spectrum betalactamase producing Klebsiella pneumoniae in neonatal intensive care unit. J Perinatol 2008; 28: 685-90.

Adams-Chapman I, and Stoll BJ. Prevention of nosocomial infections in the neonatal intensive care unit. Curr Opin Pediatr, 2002; 14: 157-64

Clinical and laboratory Standard Institute Guidelines; January 2013.

Collee JG, Miles RS, Watt B. Test for the identification of bacteria. In: Collee JG, Fraser AG, Marmion BP, Simmons A, editors. Mackie \& MacCartney practical medical microbiology, $14^{\text {th }}$ ed. Edinburgh: Churchill Livingstone; 1996. p. 151-79.

Goldmann DA, Freeman J, Durbin WA. Nosocomial infection and death in a neonatal intensive care unit. $J$ Infect Dis 1983; 147: 635-41.

Jain A and Mondal R. Prevalence \& antimicrobial resistance pattern of extended spectrum $\beta$-lactamase producing Klebsiella spp isolated from cases of neonatal septicaemia, Indian J Med Res 2007; 125: 89-94

Karunasekera KA, and Pathirana D. A preliminary study on neonatal 
septicaemia in a tertiary referral hospital paediatric unit. Ceylon Med J 1999; 44:81-6.

Lalitha MK. Manual on Antimicrobial susceptibility testing. Indian $\mathrm{J}$ of Medical Microbiologists; 2011.

Mondal GP, Raghavan M, Bhat BV and Srinivasan S. Neonatal septicaemia among inborn and outborn babies in a referral hospital, Indian J Pediatr 1991; 58: $529-533$

Report of the National Neonatal Perinatal Database (National Neonatology Forum) 2000.

Shah AJ, Mulla SA, Revdiwala SB. Neonatal sepsis: High antibiotic resistance of the bacterial pathogens in a neonatal intensive care unit of a tertiary care hospital. J Clin Neonatol 2012; 1: 72-5.

Shahid S, Malik A, Agrawal M, Singhal S, Th e phenotypic detection of the extended spectrum and the AmpC $\beta$ lactamases by a new spot inoculation method and a modified three dimensional extract test $J$ Antimicrobial Chemother 2004; 54: 684-87.

Waheed M, Laeeq A, Maqbool S. The etiology of neonatal sepsis and patterns of antibiotic resistance. J Coll Physicians Surg Pak 2003; 13: 449-52.

WHO. Perinatal mortality. Report No.: WHO/FRH/MSM/967. Geneva: WHO, 1996.

\section{How to cite this article:}

Pavneet Kaur and Sarbjeet Sharma. 2018. Bacteriological Profile and Antibiotic Sensitivity Pattern of Neonatal Sepsis in a Tertiary Care Hospital. Int.J.Curr.Microbiol.App.Sci. 7(11): 3264-3268. doi: https://doi.org/10.20546/ijcmas.2018.711.376 